\title{
Effect of a Derivative of Dithiocarbamoylhydrazine on Hormone Excretion in a Patient with Precocious Puberty
}

\author{
J. A. LORAINE,* M.B., PH.D., D.SC., F.R.C.P.ED. ; E. T. BELL, † B.SC., PH.D. ; G. L. FOSS, $\ddagger$ O.B.E., v.R.D., M.A., M.D.
}

Brit. med. F., 1965, 1, 98-99

The dithiocarbamoylhydrazine derivative, compound 33,828 (Imperial Chemical Industries Ltd.), has been shown to be an inhibitor of pituitary gonadotrophic function in rats, dogs, and monkeys (Paget et al., 1961) and of growth-promoting activity in rats (Cargill Thumpson, 1963). In post-menopausal women Bell et al. (1962a) found that compound 33,828 decreased urinary gonadotrophin excretion without affecting 17-hydroxycorticosteroid or 17-oxosteroid output; in women during reproductive life the compound was shown to be capable of inhibiting ovarian activity and ovulation, as judged by steroid excretion, without affecting gonadotrophin output (Bell et al., 1962b ; Loraine, 1964).

Because of its pituitary-inhibiting properties in human subjects and in animals it appeared possible that compound 33,828 might be of value in the treatment of sexual precocity in children. Its effects on hormone excretion in patients with this disease have not yet been investigated, and the aim of the present communication is to report such a study conducted in a girl aged 9 years suffering from precocious puberty.

Compound Administered.-Compound 33,828 is $1-\alpha-$ allylthiocarbamoyl-2-methylthiocarbamoylhydrazine

$$
\mathrm{CH}_{22}=\text { CH.CH.NH.CS.NH.NH.CS.NH.CH } \mathrm{CH}_{3}
$$

In contrast to the majority of pituitary inhibitors previously described the compound is not a steroid.

\section{Case Report}

The patient, a girl aged 9, was first noted to have sexual precocity at the age of 22 months. At that time she weighed $33 \mathrm{lb}$. (15 kg.) for her height of $35 \mathrm{in}$. $(89 \mathrm{~cm}$.). Her breasts were well developed, she showed early pubic hair and hypertrophy of the labia majora. Her skeletal age was 6 years (see Greulich and Pyle, 1959) and radiological examination of the sella turcica was negative. From the age of 2 to 9 years records were kept of her growth and development, and $x$-ray films of her left hand and wrist were taken at intervals in order to assess skeletal age. During this period her height was consistently above the 97 percentile for girls (Tanner and Whitehouse, 1959). Her growth rate was abnormally high up to the age of 6 and thereafter was below the curve for normal girls (Bayer and Bayley, 1959). Her menarche occurred at $2 \frac{1}{2}$ years; the menstrual pattern was irregular for the first year, and subsequently the cycles varied in length from 28 to 35 days. At the age of 9 she was admitted to the Bristol Children's Hospital for hormone-assay studies associated with the administration of compound 33,828 . By this time her growth had virtually ceased and her skeletal age was 16 years.

General Design of Investigation.-The patient collected 24-hour urine samples continuously throughout a period of investigation extending over 81 days. These samples were frozen and dispatched in batches by overnight train to Edinburgh. After a complete control cycle compound 33,828 was administered orally, starting on the fifth day of the next cycle (day 31 of the investigation). For the first 10

\footnotetext{
- Director, Medical Research Council Clinical Endocrinology Research Unit, University of Edinburgh.

t Member of Scientific Staff, Medical Research Council Clinical Endocrinology Research Unit, University of Edinburgh.
}

$\mp$ Clinical Assistant to Endocrine Clinics, United Bristol Hospitals. days of the treatment period the dosage was $10 \mathrm{mg} . / \mathrm{kg}$. body weight/ day given in three divided doses. During this time the patient vomited on four occasions, her appetite was poor, and pyrexia of $100^{\circ} \mathrm{F}$. $\left(37.8^{\circ} \mathrm{C}\right.$.) was present on day 37 . The dosage was therefore reduced to $7.5 \mathrm{mg} . / \mathrm{kg}$./day for the remaining 11 days of the treatment period, and during this time side-effects were absent. The administration of the compound was discontinued on day 51, and during the remainder of the study, which included a complete menstrual cycle, no further treatment was given. Throughout the period of investigation episodes of bleeding and rectal basal temperatures were recorded.

Assay Methods.-Human pituitary gonadotrophins (H.P.G.) were measured by the method of Loraine and Brown (1959). The results were expressed in terms of the International Reference Preparation for Human Menopausal Gonadotrophin as H.M.G. units per 24hour urine sample. Urinary oestriol, oestrone, and oestradiol were determined by the method of Brown (1955) as modified by Brown et al. (1957). Pregnanediol and pregnanetriol were estimated by the methods of Klopper et al. (1955) and Fotherby and Love (1960) respectively. For the estimation of 17-hydroxycorticosteroids and total 17-oxosteroids, modifications of the techniques described by Vestergaard (1951) and Appleby et al. (1955) were employed. Dehydroepiandrosterone was measured by the method of Fotherby (1959).

\section{Results of Investigations}

The results are shown in the Chart. The first cycle was probably of an ovulatory character as judged by the pattern of oestrogen excretion in urine: however, levels for all three oestrogens were in general lower than those found by Loraine and Bell (1963) in a series of 15 ovulatory menstrual cycles. Pregnanetriol excretion was low on days 1 to 6 and 11 and 12 of the investigation; during the remainder of the cycle values were higher and relatively constant. The pattern of pregnanediol excretion was atypical (see Loraine and Bell, 1963): a marked luteal phase rise did not occur, but instead the level gradually increased from days 17 to 26 of the study and fell rapidly at the time of menstruation. Throughout this cycle H.P.G.excretion values were at the lower end of the normal range and an ovulatory peak at mid-cycle did not occur.

During the 21 days of drug administration the urinary excretion of oestrogens and pregnanediol remained uniformly low, indicating that ovarian activity and ovulation had been suppressed. Pregnanetriol output was little affected, suggesting that the precursors of this steroid were derived from the adrenal cortex rather than from the ovary. The compound had no significant effect on H.P.G. excretion as compared with the control cycle, but it should be noted that the mean H.P.G. level was significantly lower in the first nine days as compared with the last 11 days of the treatment period $(0.01>\mathrm{P}>0.001)$.

After withdrawal of the compound on day 51 of the study menstruation did not occur for a further 28 days. During this time urinary oestrogen levels rose to an ovulatory peak on days 63 and 64 , and this was followed by a luteal phase rise in the excretion of oestrogens, pregnanediol, and pregnanetriol. The mean H.P.G. output in this cycle did not differ significantly from that in the control cycle or in the period of drug administration.

No alteration in the excretion of total 17-oxosteroids, 17-hydroxycorticosteroids, and dehydroepiandrosterone was produced by the administration of compound 33,828. The levels for total 17-oxosteroids were within the normal range throughout the period 17-oxost (see Hamburger, 1948). Dehydroepiandrosterone excretion was generally so low that the levels could not be detected by the method in use. Values for 17-hydroxycorticosteroids fluctuated from 
5.1 to $11.5 \mathrm{mg} . / 24$ hours at various stages of the investigation; some of these readings were abnormally high for a child of this age (see Borth et al., 1957). Basal temperature records showed the presence of a monophasic curve in the first cycle and a biphasic curve in the second.

\section{Discussion}

The effects of compound 33,828 on hormone excretion in the child with precocious puberty were very similar to those previously reported by Bell et al. (1962b) in normally menstruating women. Ovarian activity and ovulation were suppressed during administration of the compound, while inhibition of pituitary function as judged by urinary H.P.G. assays did not occur. As in the previous study, a differential inhibition of either follicle-stimulating hormone or luteinizing hormone could not be excluded because the bioassay method used for H.P.G., the mouse-uterus test, measures a mixture of these two activities (SchmidtElmendorff et al., 1962). In normally menstruating women and in the subject reported above menstruation did not occur after withdrawal of the compound. With oral progestational agents, on the other hand, bleeding generally takes place a few days after they are discontinued.

During the period of drug administration the mean H.P.G. excretion was significantly lower when the higher dosage of compound 33,828 was administered. This finding is in agreement with that already reported by Bell et al. (1962a) in postmenopausal women, in whom it has been shown that the degree of pituitary inhibition was directly related to the dosage of the compound employed. In the present study the compound produced no effect on adrenocortical function as judged by assays of urinary 17-hydroxycorticosteroids and 17-oxosteroids, an observation already made in post-menopausal subjects so treated.

\section{Summary}

The effect of the dithiocarbamoylhydrazine derivative, compound 33,828 (I.C.I.), on pituitary, ovarian, and adrenal function was studied in a 9-year-old patient with precocious puberty and with a history of menstrual cycles of variable length beginning at the age of $2 \frac{1}{2}$ years.

The compound suppressed ovarian activity and ovulation as judged by steroid assays in urine. The excretion of human pituitary gonadotrophins was little affected.

Compound 33,828 produced no effect on adrenocortical function as estimated by assays of urinary 17-hydroxycorticosteroids and 17-oxosteroids.

In the performance of the assays the skilled assistance of many technicians at the Clinical Endocrinology Research Unit, and in particular of Mr. H. A. F. Blair and Mr. D. N. Love, was much appreciated. Thanks are due to Imperial Chemical Industries Ltd. for supplies of compound 33,828. We are grateful to Professor
A. V. Neale for admitting this patient to his wards during the period of investigation.

\section{REFERENCES} Appleby, J. I., Gibson, G., Norymberski, J. K., and Stubbs, R. D. (1955).
Biochem. F., 60, 453.

Bayer, L. M., and Bayley, N. (1959). Growth Diagnosis. University of

Bell, E. T., Brown, J. B., Fotherby, K., Loraine, J. A., and Robson, J. S. (1962a). F. Endocr., 25, 221.

Borth, R., Linder, A., and Riondel, Lancet, 2, 528.

33.

Brown, J. B. (1955). Biochem. F., 60, 185.

- Bulbrook, R. D., and Greenwood, F. C. (1957). F. Endocr., 16,

argill

Fotherby, K. (1959). Biochem. F., 73, 339.

- and Love, D. N. (1960). F.' Endocr., 20, 157

Greulich, W. W., and Pyle, S. I. (1959). Radiographic Atlas of Skeletal Development of the Hand and Wrist, 2nd ed. Oxford University Press, London.

Hamburger, C. (1948). Acta endocr. (Kbh.), 1, 19. Klopper, A., Michie, E. A., and Brown, J. B. (1955). F. Endocr., 12,

Loraine, J. A. (1964). Int. 7. Fertil., 9, 155.

- and Bell, E. T. (1963). Lancet, 1, 1340.

- and Brown, J. B. (1959). F. Endocr., 18, 77.

Paget, G. E., Walpole, A. L., and Richardson, D. N. (1961). Nature (Lond.), 192, 1191 .

Schmidt-Elmendorff, H., Loraine, J. A., and Bell, E. T. (1962). F. Endocr., 24, 349

Tanner, J. M., and Whitehouse, R. H. (1959). Institute of Child Health Charts Prepared for the Hospital for Sick Children, Great Ormond Street, London. Joseph Collard, London.

Vestergaard, P. (1951). Acta endocr. (Kbh.), 8, 193. 